\title{
Integrated Turbine Tip Clearance and Gas Turbine Engine Simulation
}

\author{
Jeffryes W. Chapman* \\ Vantage Partners, LLC, Brook Park, OH, 44142, USA \\ Jonathan Kratz, ${ }^{\dagger}$ Ten-Huei Guo, ${ }^{\ddagger}$ and Jonathan Litt ${ }^{\S}$ \\ NASA Glenn Research Center, Cleveland, OH, 44135, USA
}

\begin{abstract}
Gas turbine compressor and turbine blade tip clearance (i.e., the radial distance between the blade tip of an axial compressor or turbine and the containment structure) is a major contributing factor to gas path sealing, and can significantly affect engine efficiency and operational temperature. This paper details the creation of a generic but realistic high pressure turbine tip clearance model that may be used to facilitate active tip clearance control system research. This model uses a first principles approach to approximate thermal and mechanical deformations of the turbine system, taking into account the rotor, shroud, and blade tip components. Validation of the tip clearance model shows that the results are realistic and reflect values found in literature. In addition, this model has been integrated with a gas turbine engine simulation, creating a platform to explore engine performance as tip clearance is adjusted. Results from the integrated model explore the effects of tip clearance on engine operation and highlight advantages of tip clearance management.
\end{abstract}

$\begin{array}{ll}\text { C-MAPSS40k } & \text { Commercial Modular Aero-Propulsion System Simulation 40k } \\ \text { EGT } & \text { Exhaust Gas Temperature } \\ \text { EPR } & \text { Engine Pressure Ratio } \\ \text { FADEC } & \text { Full Authority Digital Engine Control } \\ \text { HPC } & \text { High Pressure Compressor } \\ \text { HPT } & \text { High Pressure Turbine } \\ \text { LPC } & \text { Low Pressure Compressor } \\ \text { SFC } & \text { Specific Fuel Consumption } \\ \text { SLS } & \text { Sea Level Static } \\ \text { TCC } & \text { Tip Clearance Control } \\ \text { T-MATS } & \text { Toolbox for the Modeling and Analysis of Thermodynamic Systems } \\ \mathrm{ft} & \text { Feet } \\ \text { mils } & \text { thousands of an inch } \\ { }^{\circ} \mathrm{R} & \text { Degrees Rankine } \\ \mathrm{rpm} & \text { Revolutions per minute } \\ \mathrm{S} & \text { Seconds } \\ A & \text { Area } \\ \text { Bi } & \text { Biot number } \\ C_{p} & \text { Specific heat } \\ E & \text { Modulus of elasticity } \\ \text { Fo } & \text { Fourier number } \\ \end{array}$

\footnotetext{
* Aerospace Engineer, 3000 Aerospace Parkway, Brook Park OH, AIAA Member.

${ }^{\dagger}$ Research Engineer, 21000 Brookpark Rd., Cleveland OH, MS-77-1, AIAA Member.

‡ Research Engineer, 21000 Brookpark Rd., Cleveland OH, MS-77-1, AIAA Member.

$\S$ Research Engineer, 21000 Brookpark Rd., Cleveland OH, MS-77-1, AIAA Senior Member.
} 


\begin{tabular}{ll} 
Fnet & Net thrust \\
$K$ & tip clearance multiplier \\
$L$ & Length \\
$N$ & Shaft speed \\
$N C$ & Engine core speed \\
$N f$ & Engine fan speed \\
$P s 3$ & Static pressure at engine station 3 \\
$Q$ & Energy \\
s_Eff_TC & HPC efficiency scalar based on tip clearance \\
$T$ & Temperature \\
$T C$ & Tip clearance \\
$V o l$ & Volume \\
$\Delta$ & Change in \\
$h$ & Convection coefficient \\
$k$ & Conduction coefficient \\
$p$ & Discrete time step \\
$r$ & Radius \\
$t$ & Time \\
$u$ & Partial deflection \\
$x$ & Thickness \\
$\alpha$ & Expansion coefficient \\
$\partial$ & Differential \\
$\delta$ & Deflection \\
$\eta$ & Film cooling coefficient \\
$\rho$ & Density \\
$v$ & Poisson's ratio \\
$\psi_{\text {Ztip }}$ & Zweifel loading coefficient \\
& \\
$a$ & \\
avg & Type designator \\
$b$ & Average \\
$i$ & Type designator \\
$m$ & Numerator \\
$n$ & Node number adder \\
$p$ & Node number \\
$r e f$ & Time step \\
tran & Reference \\
$\infty$ & Transition \\
& \\
& Infinity \\
& \\
\hline &
\end{tabular}

\section{Introduction}

CAS turbine blade tip sealing for axial turbines has been a major concern in aircraft engine design due to the direct

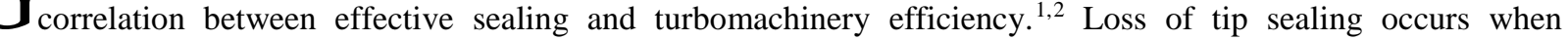
component sizes change due to thermal or mechanical load transients applied to the engine structures, or when blades or the abradable tip seal deteriorate. Maintaining a tip clearance that is both small enough to effectively seal and also large enough to reduce the possibility of the rotating blade rubbing the static structure is a design objective during engine operation. Because tip clearance changes during engine operation, active tip clearance control is a current area of research. ${ }^{3}$ Simulation of tip clearance for a specific engine can be very complicated, as tip clearance is affected by cooling and gas path flow. High fidelity models of these phenomena many times require an in-depth understanding of component geometry and rely on resource intensive computational fluid dynamics methods. A generic model that uses geometric assumptions and idealized flow interaction can be a much simpler alternative when a high fidelity, engine specific simulation is not required. This paper will explore the second case for the purpose of conducting active tip clearance control (TCC) research. It expands on the modeling effort done by Kypuros and Melcher, ${ }^{4}$ and details the 
creation of a generic tip clearance model of appropriate fidelity, fully integrated with a generic gas turbine engine model.

The benefits of optimizing turbine tip clearance during aircraft operation is well documented. Holeski and Futral ${ }^{5}$ experimentally found that an increase in tip clearance directly caused a drop in turbine efficiency. This change in efficiency can have an effect on the total mission specific fuel consumption (SFC), as Wiseman and Guo ${ }^{6}$ found that a 10 mil reduction of clearance can result in an overall efficiency improvement of as much as $1 \%$ over the course of a mission for a common gas turbine engine. Additionally, reduction in tip clearance lowers operating temperatures as the increase in efficiency allows the engine to run at a given point with less fuel burn, as shown by Bringhenti and Barbosa. ${ }^{7}$ The new lower operating temperature results in less engine deterioration, which increases time-on-wing, and lowers maintenance cost.

Turbine tip clearance can generally be defined as the distance between the turbine blade tip and the turbine shroud or turbine containment structure, as shown in Fig. 1. High pressure turbine (HPT) tip clearance is typically designed with two main objectives, maintain a steady-state clearance and minimize pinch points during operation. During engine transients such as take-off, increases in shaft speed result in blade and rotor growth relative to the case. These thermal growth phenomena can cause pinch points. Once heat begins to soak into the case, rotor, and blade, the clearance begins to widen until a steady-state tip clearance is reached. To reduce the chance of blades rubbing against the shroud, the engine clearance must be designed around these pinch points, which can have a detrimental effect on the abradable tip seal and the resulting tip clearance at steady-state.

Interest in tip clearance control research has intensified as requirements for higher efficiency engines have increased. These control methods have typically taken the form of active or passive thermal, mechanical, or pneumatic systems. Thermal control systems make use of engine bypass or bleed air to manage the size of components with heating or cooling, mechanical control designs utilize external actuation to vary tip clearance, ${ }^{8}$ and pneumatic control systems make use of engine generated pressures to maintain clearance levels. To facilitate development of these types of systems, a sensitivity study will be performed relating system characteristics with tip clearance magnitude and engine performance.

Simulation updates described in this paper are applied to the Commercial Modular Aero-Propulsion System Simulation 40k ${ }^{9}$ (C-MAPSS40k), shown in the boxed region of Fig. 2. C-MAPSS40k is a nonlinear dynamic model of a generic, high-bypass, dual-spool turbofan engine with a thrust capacity of $40,000 \mathrm{lb}$. Written in a combination of MATLAB/Simulink and the $C$ programming language, the engine simulation offers two main components, a high fidelity engine model and a control system representative of a Full Authority Digital Engine Controller (FADEC). ${ }^{10}$ The engine model simulation uses a combination of physics-based energy balance equations and empirical compressor and turbine performance maps to generate realistic engine data. The C-MAPSS40k control logic is based on a gain scheduled proportional-integral controller that uses fuel flow to drive the engine to a specified engine pressure ratio (EPR), which is directly correlated to thrust. Safe operation is governed by sets of limiters (such as shaft speed and acceleration limiters) that maintain engine variables within acceptable boundaries.

Baseline C-MAPSS40k does not simulate HPT tip clearance, so the custom tip clearance model developed in this paper is integrated with the simulation by utilizing a subset of C-MAPSS40k generated parameters, such as cooling flow and core flow temperatures and pressures. Parameters of interest for the modeling effort include cooling flow temperature, turbine input and output temperatures, and shaft speed.

Once tip clearance modeling accuracy is verified, the model is integrated into the C-MAPSS40k platform by connecting the inputs as described above and establishing an additional efficiency scalar into the HPT, as shown in Fig. 2. To generate this scalar, tip clearance will be determined by running the engine with a generic passive type tip clearance scheme. The average value of the tip clearance for this passive scheme is considered the nominal value and coincides with no change in turbine efficiency. Once the nominal tip clearance is established, off nominal values of clearance will be assigned varying degrees of efficiency change, as described later in the paper. 


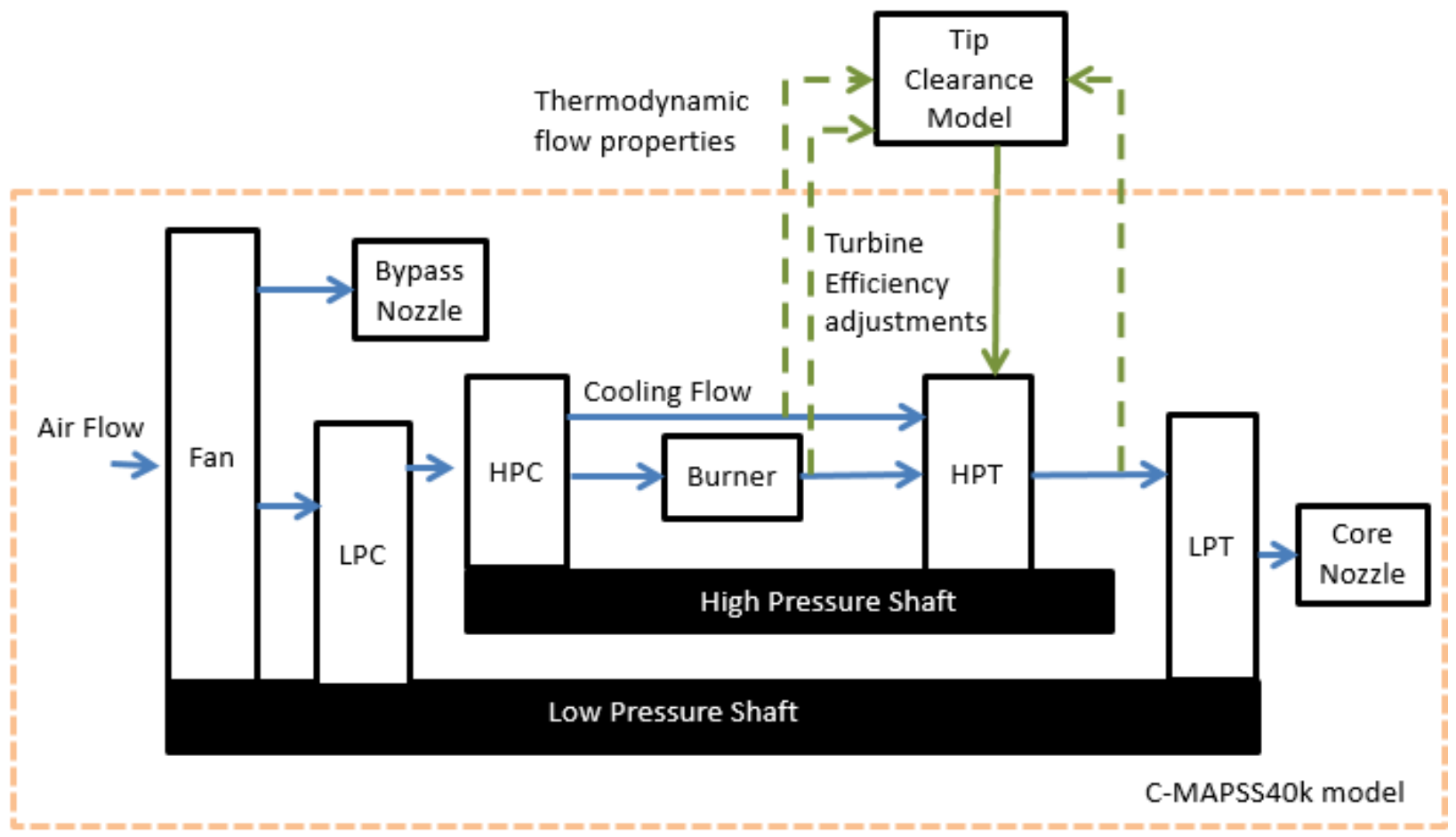

Figure 2. Tip clearance control model integrated with C-MAPSS40k engine model.

Subsequent sections of this paper detail the development of the tip clearance model and its integration with a generic engine simulation, study effector sensitivity, and discussion of future work. Specifically, development and implementation of the tip clearance model is discussed in Section II, followed by a discussion of engine model integration in Section III. Validation of the total model is in Section IV, an input sensitivity study appears in Section V, and a summary of future work is in Section VI. Finally, conclusions are given in Section VII.

\section{Tip Clearance Modeling}

The tip clearance modeling strategy discussed in this paper was leveraged from previous work done by Kypuros and Melcher. ${ }^{4}$ In that work, the HPT was assumed to contain three distinct physical members: rotor, blade, and shroud. Here, the shroud has distinct outer and inner layers to distinguish between the superalloy structure and its abradable thermal coating, respectively. The HPT tip clearance gap (TC) was defined as the distance between the blade tip and the shroud, and is a function of numerous factors including shaft speed, shroud cooling air, core engine flow, rotor cooling flow, and the component dimensions. A simple diagram of the tip clearance system is shown in Fig. 3. The $T C$ is modeled as a function of nominal clearance $\left(T C_{\text {nominal }}\right)$ and the net mechanical deformation (in the radial direction) of the HPT shroud, rotor, and blade, shown as $\delta_{\text {shroud }}, \delta_{\text {rotor }}$, and $\delta_{\text {blade }}$ respectively, in Eq. (1). This equation may also be formulated as a function of shroud inner radius $\left(r_{\text {shroud }}\right)$, rotor radius $\left(r_{\text {rotor }}\right)$, and blade length $\left(L_{\text {blade }}\right)$.

$$
\begin{gathered}
T C(t)=T C_{\text {nominal }}+\left(\delta_{\text {shroud }}-\delta_{\text {rotor }}-\delta_{\text {blade }}\right) \\
T C_{\text {nominal }}=r_{\text {shroud }}-\left(r_{\text {rotor }}+L_{\text {blade }}\right)
\end{gathered}
$$

where each of these variables are dependent on engine operating condition.

Calculations of individual deformations are based on component temperatures and, for the rotor and blades, centrifugal forces that are updated as the engine operates. This modeling includes deformation components that are both elastic and axisymmetric. Plastic and asymmetric deformation modeling is beyond the scope of this paper and will not be discussed. Updates to the models discussed in Ref. 4 are limited to adjusting the transient heat transfer equations. These equations from Ref. 4 rely on assumptions based on a simplified step input in temperature, and are replaced with state based heat equations that use finite difference methods. This adjustment increases accuracy when component temperature states are updated continuously. The following sub-sections detail the tip clearance model and 
contain a full description of the heat transfer updates as well as a summary of the deflection equations. Turbine baseline properties, such as geometry and material properties, were obtained directly from Ref. 4 and will not be detailed here.

\section{Shroud cooling air}

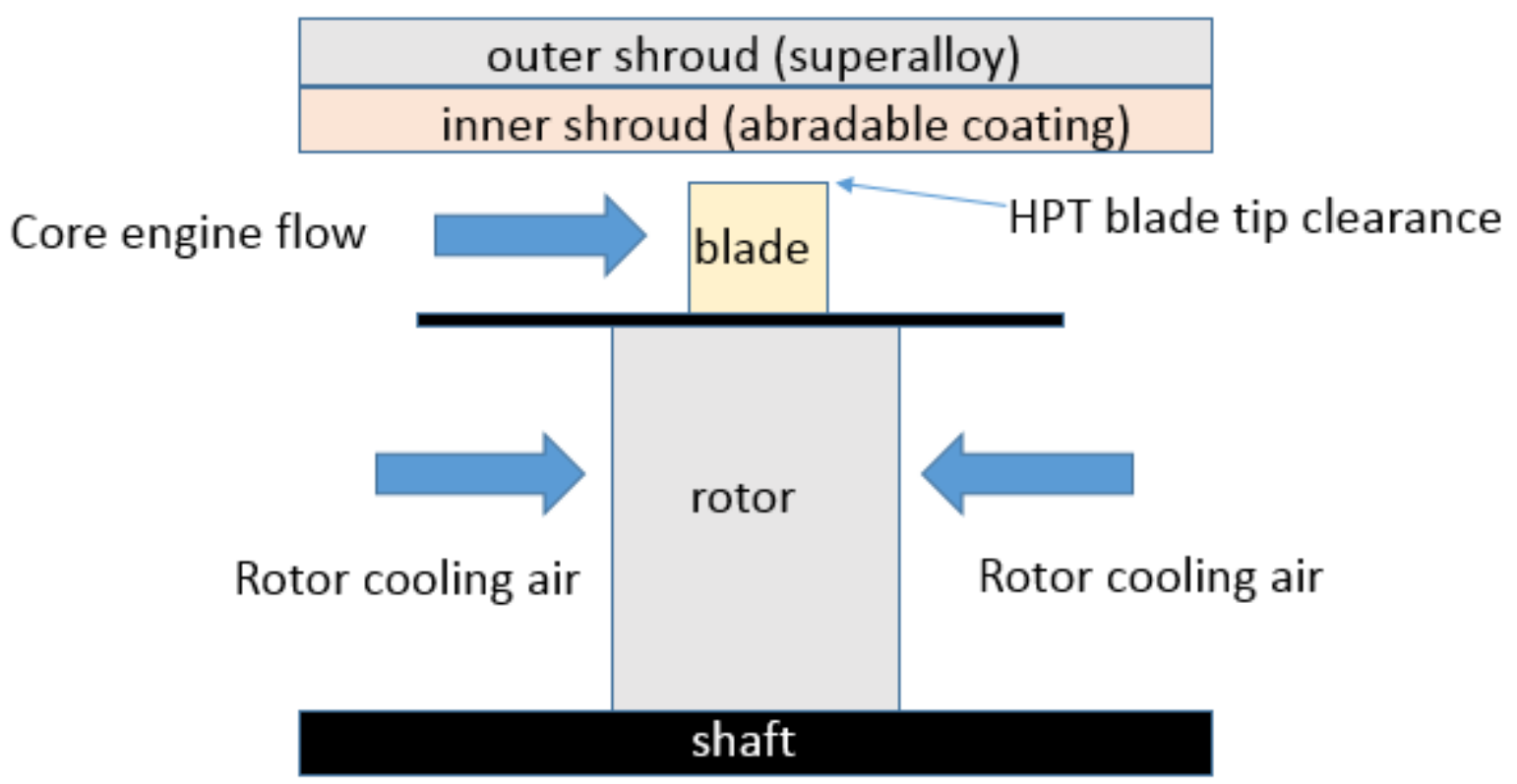

Figure 3. Diagram of assumed HPT components.

\section{A. Shroud}

The shroud is a cylindrical containment structure composed of a superalloy material that encases the HPT flow path. Its interior walls (facing the HPT flow path) are covered by a thin layer of an abradable material which acts as a thermal barrier. The shroud is built to withstand the high temperatures of the turbine flow path on the interior wall while the exterior is cooled with high pressure compressor (HPC) bypass flow. For this paper, it is assumed that deformation of the shroud is a result of the thermal states of the gases in contact with the shroud surfaces, and is not affected by outside structures such as the casing and/or other structural supports.

Thermal modeling of the shroud makes use of the heat equation with uniform conductive and convective surfaces, and without internal heat generation. ${ }^{11} \mathrm{~A}$ simplified 1-D plate form (rather than a cylinder) was assumed based on the shroud's thin material width relative to its radius. The heat equation is shown in Eq. (2), where $T$ is temperature, $x$ is thickness, $t$ is time, $\rho$ is density, $k$ is the conduction coefficient, and $C_{p}$ is specific heat.

$$
\frac{\rho * C_{p}}{k} * \frac{\partial T}{\partial t}=\frac{\partial^{2} T}{\partial x^{2}}
$$

The shroud consists of two materials with combustor air in contact with the interior surface and cooling air in contact with the exterior surface. To simplify the model, the heat equation is discretized using the Crank-Nicolson method, ${ }^{12}$ then each material is divided into evenly spaced temperature states, or nodes, as shown in Fig. 4 , where $\partial x_{a}$ and $\partial x_{b}$ are the node thicknesses of the different materials, $T_{\text {turbine }}$ is the average turbine flow path temperature, $T_{\text {cool }}$ is the temperature of the cooling bleed, and the $T_{i}$ values are the node temperature states. Although evenly spaced radially, nodes on the surface of a material are half the width of interior nodes. 


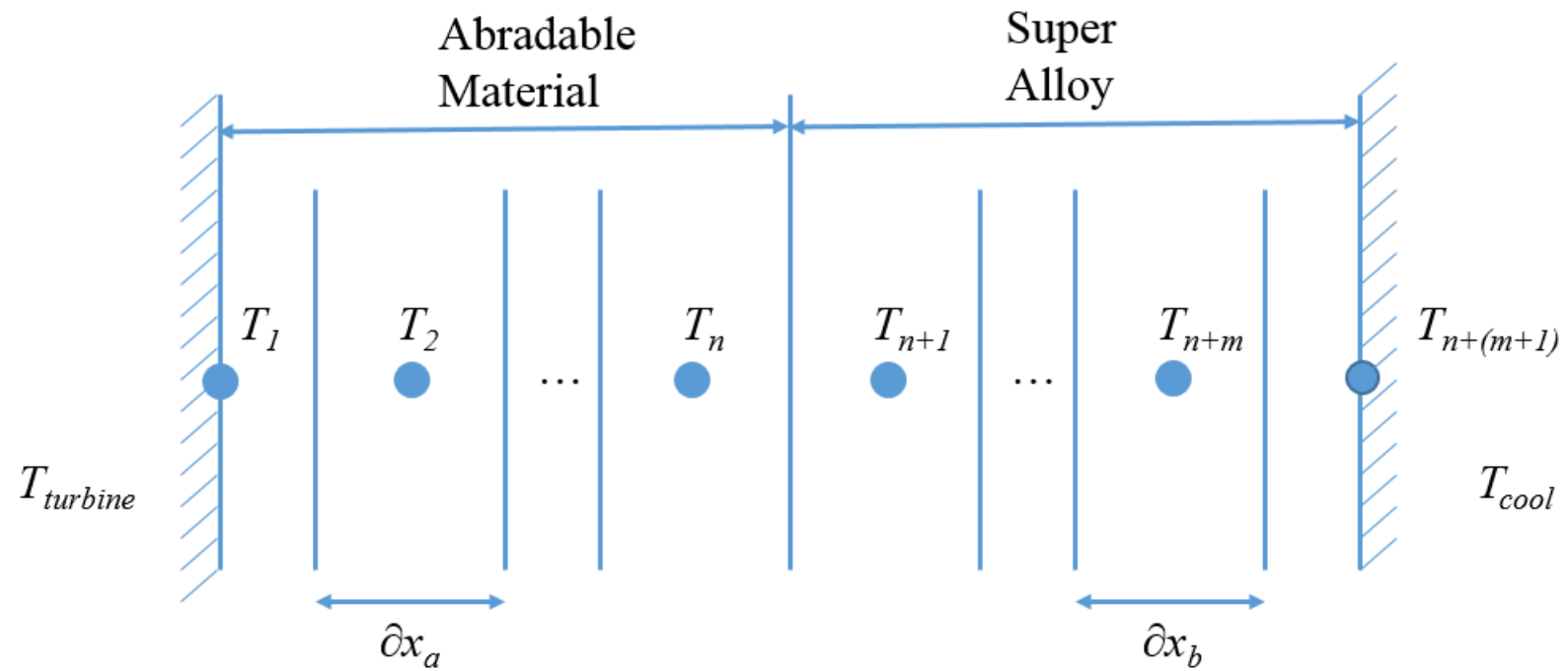

Figure 4. Shroud 1-D heat transfer representation.

Changes in node temperature are determined by the energy transfer between adjacent materials and are governed by conduction when the adjacent material is a solid, or convection when the adjacent material is a fluid. Interaction between nodes is derived from the heat equation and the Crank-Nicolson method. The Crank-Nicolson method utilizes the average of the forward and backward finite difference methods used to numerically solve partial differential equations, and has the advantages of high accuracy with high numerical stability. The model operates by providing temperature states at the current time step $(p)$, then calculating the current temperature using a guessed future $(p+1)$ temperature and the current temperature. This calculated current temperature is then compared with the actual current temperature to create a temperature error that is subsequently used to tune the future guess value to the actual future value. Tuning is performed using a Newton-Raphson solver leveraged from the Toolbox for the Modeling and Analysis of Thermodynamic Systems (T-MATS), a Simulink add-on containing blocks useful for thermodynamic simulation. ${ }^{13}$ Generalized equations developed for the shroud temperature model are laid out as follows:

General node energy equation:

$$
\dot{Q}_{\text {node }}=\sum \dot{Q}_{\text {conduction }}+\sum \dot{Q}_{\text {convection }}
$$

and:

$$
\begin{gathered}
\dot{Q}_{\text {node }}=\frac{\rho * \mathrm{Vol} * C_{p}}{\partial t}\left(T_{n}^{p+1}-T_{n}^{p}\right) \\
\dot{Q}_{\text {conduction }}=\frac{k * A}{2 * \partial x}\left(\left(T_{n \pm 1}^{p}-T_{n}^{p}\right)+\left(T_{n \pm 1}^{p+1}-T_{n}^{p+1}\right)\right) \\
\dot{Q}_{\text {covection }}=\frac{h * A}{2}\left(\left(T_{n \mp 1}^{p}-T_{n}^{p}\right)+\left(T_{n \mp 1}^{p+1}-T_{n}^{p+1}\right)\right)
\end{gathered}
$$

where, $Q$ is energy, $\rho$ is density, $\mathrm{Vol}$ is volume, $C_{p}$ is specific heat, $\partial t$ is change in time, $\partial x$ is change in material width, $A$ is surface area, $h$ is the convection coefficient, $k$ is the convection coefficient, and $T$ is temperature. Note, $T$ subscripts denote temperature node states and $T$ postscripts denote temperature time steps. Equation (3) is then formulated for the general use cases below, based on node location within the material.

Exterior or edge (Convective) node, $T_{1}$ and $T_{n+(m+1)}$ :

$$
\begin{aligned}
T_{\text {edge }}^{p}= & F o(1-B i) *\left(T_{\text {edge }}^{p+1}+T_{\text {edge }}^{p}\right) \\
& -F o\left(T_{\text {interior }}^{p+1}+T_{\text {interior }}^{p}+B i\left(T_{\infty}^{p+1}+T_{\infty}^{p}\right)\right)+T_{\text {edge }}^{p+1}
\end{aligned}
$$

where $F o$ is the Fourier number for the material, $B i$ is the Biot number for the convective exchange, $T_{\text {edge }}$ is the temperature of the edge node, $T_{\text {interior }}$ is the temperature of the adjacent node in the material, and $T_{\infty}$ is the temperature 
of the adjacent fluid ( $T_{\text {turbine }}$ or $T_{\text {cool }}$ in Fig. 4). For convenience, equations for Biot number and Fourier number are shown in Eq. (4).

$$
\begin{gathered}
F o=\frac{\Delta t * k}{\rho * C_{p} * \partial x^{2}} \\
B i=\frac{h * \partial x}{k}
\end{gathered}
$$

Interior (Conductive) nodes within the abradable material or superalloy:

$$
T_{n}^{p}=F_{O} *\left(T_{n}^{p}+T_{n}^{p+1}\right)-\frac{F o}{2}\left(T_{n-1}^{p}+T_{n-1}^{p+1}+T_{n+1}^{p}+T_{n+1}^{p+1}\right)+T_{n}^{p+1}
$$

Interface (Conductive) node, $T_{n}$ and $T_{n+1}$ :

$$
\begin{aligned}
T_{n}^{p}= & \frac{F o_{\text {node }}+F o_{\text {trans }}}{2}\left(T_{n}^{p}+T_{n}^{p+1}\right)-\frac{F o_{\text {node }}}{2}\left(T_{n \pm 1}^{p}+T_{n \pm 1}^{p+1}\right) \\
& -\frac{F o_{\text {trans }}}{2}\left(T_{n \mp 1}^{p}+T_{n \mp 1}^{p+1}\right)+T_{n}^{p+1}
\end{aligned}
$$

where $F o_{\text {node }}$ is the Fourier number for the node material as given in Eq. (4), and $F o_{\text {trans }}$ is the transition Fourier number. The $\mathrm{Fo}_{\text {trans }}$ for a transition between node material $a$ and material $b$, would appear as in Eq.(7).

$$
F o_{\text {trans, a to } b}=\frac{\Delta t *\left(\frac{\partial x_{a} * k_{a}+\partial x_{b} * k_{b}}{\partial x_{a}+\partial x_{b}}\right)}{\rho_{a} C_{p, a} \partial x_{a}\left(\frac{\partial x_{a}+\partial x_{b}}{2}\right)}
$$

where material property constants with subscript $a$ are for node material $a$, and constants with subscript $b$ are for material $b$.

Shroud thermal deformation equations were leveraged from Kypuros ${ }^{4}$ and are summarized below for convenience:

$$
\delta_{\text {shroud }}=\alpha_{\text {shroud }} * r_{\text {shroud }} * T_{\text {avg }}
$$

where $\delta_{\text {shroud }}$ is shroud deflection, $r_{\text {shroud }}$ is the inner radius of the superalloy portion of the shroud, $\alpha_{\text {shroud }}$ is shroud expansion coefficient, and $T_{a v g}$ is the mean temperature of the shroud derived from temperature states calculated with Eqs. (3) to (6). It should be noted that the abradable material is assumed to have no effect on the deflection of the shroud and is considered a simple coating that has no structural influence on the system.

\section{B. Rotor}

The rotor is defined, for the purposes of this paper, as the structure connecting the turbine blades to the shaft. Modeling of this component follows the assumptions detailed in Ref. 4 where the rotor is taken to be a disk that undergoes deflection caused by temperature changes on each side of the disk and centrifugal forces. Heat transfer through the structure is determined by the 1-D finite-difference method detailed in Eqs. (3) and (5), with 20 distinct nodes and surface fluid temperatures equal to the HPC bleed cooling flow.

Rotor thermal and centrifugal deformation equations are based on elastic material properties and are summarized below:

$$
\begin{gathered}
\delta_{\text {rotor }}=u_{\text {rotor } 1}+u_{\text {rotor } 2} \\
u_{\text {rotor } 1}=\alpha_{\text {rotor }} * r_{\text {rotor }} * T_{\text {avg }} \\
u_{\text {rotor } 2}=\frac{1}{4 E_{\text {rotor }}}\left(1-v_{\text {rotor }}\right) \rho_{\text {rotor }} * r_{\text {rotor }}^{3} *\left(\frac{2 \pi}{60} N\right)^{2}
\end{gathered}
$$

where $\delta_{\text {rotor }}$ is total rotor deflection, $u_{\text {rotor } 1}$ is deflection due to change in temperature, $u_{\text {rotor } 2}$ is deflection due to centrifugal forces, $\alpha_{\text {rotor }}$ is the rotor expansion coefficient, $r_{\text {rotor }}$ is the rotor radius, $T_{\text {avg }}$ is the mean temperature of the rotor, $E_{\text {rotor }}$ is the modulus of elasticity for the rotor, $v_{\text {rotor }}$ is Poisson's ratio for the rotor, $\rho_{\text {rotor }}$ is the rotor density, and $N$ is shaft speed. 


\section{Blades}

HPT turbine blades are the small section of material used to gather power from the high temperature gas stream. Deflection within the blade is determined based on thermal and centrifugal effects. The low ratio of volume to surface area allows the blade to be treated as a single mass for heat transfer modeling purposes, with surrounding fluidic temperatures a function of the turbine flow path temperature and blade cooling flow temperature. It has also been assumed that the centrifugal force is mainly a function of shaft speed and does not vary significantly as a function of rotor or blade growth. Development of the deflection equations, detailed in Ref. 4, and lumped system heat transfer equations, detailed in Ref. 11, are summarized in the Eqs. below:

$$
\begin{gathered}
\delta_{\text {blade }}=u_{\text {blade } 1}+u_{\text {blade } 2} \\
u_{\text {blade } 1}=\alpha_{\text {blade }} * L_{\text {blade }} * T_{\text {blade }} \\
T_{\text {blade }}^{p+1}=\left(T_{\text {blade }}^{p}-T_{\text {ref }}\right) * e^{-\frac{h_{\text {blade }} A_{\text {blade }}}{\rho_{\text {blade }} * \text { Vol } l_{\text {blade }} * C_{p \text { blade }}} \partial t}+T_{\text {ref }} \\
T_{\text {ref }}=\eta\left(T_{\text {cool }}-T_{\text {turb }}\right)+T_{\text {turb }} \\
u_{\text {blade } 2}=\frac{\rho_{\text {blade }} L_{\text {blade }}^{2}\left(L_{\text {blade }}+r_{\text {rotor }}\right)}{E_{\text {blade }}}\left(\frac{2 \pi}{60} N\right)^{2}
\end{gathered}
$$

where $\delta_{\text {blade }}$ is total blade deflection, $u_{\text {blade } 1}$ is deflection due to thermal effects, $u_{\text {blade2 }}$ is deflection due to centrifugal forces, $\alpha_{\text {blade }}$ is the blade expansion coefficient, $L_{\text {blade }}$ is the blade length, $T_{\text {blade }}$ is blade temperature, $T_{r e f}$ is blade reference temperature, $h_{\text {blade }}$ is the convection heat transfer coefficient, $A_{\text {blade }}$ is the blade surface area, $\rho_{\text {blade }}$ is the blade material density, $V_{o l} l_{\text {blade }}$ is the blade volume, $C_{p, \text { blade }}$ is the specific heat of the blade, $\partial t$ is the time step, $\eta$, is the film cooling efficiency, $T_{\text {cool }}$ is the cooling flow temperature, $T_{\text {turb }}$ is the mean turbine gas path temperature, $r_{\text {rotor }}$ is the rotor radius, $E_{\text {blade }}$ is the modulus of elasticity of the blade, and $N$ is the shaft speed.

\section{Effects of Tip Clearance on Turbine Performance}

As mentioned above, the tip clearance model was integrated with the overall engine model by implementing a scalar that was used to adjust the efficiency of the turbine based on the change in tip clearance over the course of a mission. This turbine efficiency scalar (s_Eff_TC) was approximated using a simple efficiency loss model suggested by Baskharone ${ }^{14}$ that utilizes relevant geometrical parameters, as shown in Eq. (11). This tip clearance efficiency model was tuned using experimental results found in Ref. 5. In these experiments, Holeski and Futral measured effective efficiency of a turbine as they manually varied the tip clearance. Results from Ref. 5 show that a 6.8\% increase in tip clearance to passage height correlates to a $20 \%$ efficiency loss.

$$
S_{-} E f f_{-} T C=1-K\left(\frac{T C-T C_{\text {baseline }}}{L_{\text {blade }}}\right)\left(\frac{r_{\text {shroud }}}{\frac{r_{\text {rotor }}+r_{\text {shroud }}}{2}}\right)
$$

Where $K$ is defined as:

$$
K=1+0.586\left(\psi_{\text {Ztip }}^{3.63}\right)
$$

and $\psi_{\text {Ztip }}$ is the Zweifel loading coefficient.

\section{Model Validation}

The tip clearance model validation was broken into sections: validation of component temperature calculations, deflections, and engine performance. Tip clearance modeling parameters were taken from Ref. 4, with the inner shroud, outer shroud, and rotor comprised of 20 distinct nodes. Temperatures and speeds from C-MAPSS40k were fed to the tip clearance model with a cooling flow assumed to come from the exit of the HPC. Resulting deflection, 
temperature transient profiles, temperature averages, and engine performance were compared for relative and absolute plausibility based on results presented in literature.

To demonstrate tip clearance model performance, an engine transient consisting of a step request of ground idle to max power was performed, as shown in Fig. 5. The resulting transient produced the following inputs to the tip clearance model: shaft speed increases from 9200 to 11900 revolutions per minute (rpm), turbine temperature increases from 1500 to 2500 degrees Rankine $\left({ }^{\circ} \mathrm{R}\right)$, and compressor temperature increases from 1000 to $1480{ }^{\circ} \mathrm{R}$. Looking at resulting temperature transients, it can be seen that the blade has the fastest response, followed by the shroud, and then the rotor. Deflections for each component generally follow the form of the temperature transients, however rotor deflection jumps initially as the shaft speed is increased. Once the rotor begins to heat, the deflection due to temperature begins to eclipse the speed effect. Clearance can be seen to follow both overall trends, as the initial transients are dominated by the fast time constants attributed to centrifugal forces (blade and rotor) and small body temperature soak (blade), and the steady state response is dominated by the slow time constants related to the large body temperature soak within the rotor and shroud.
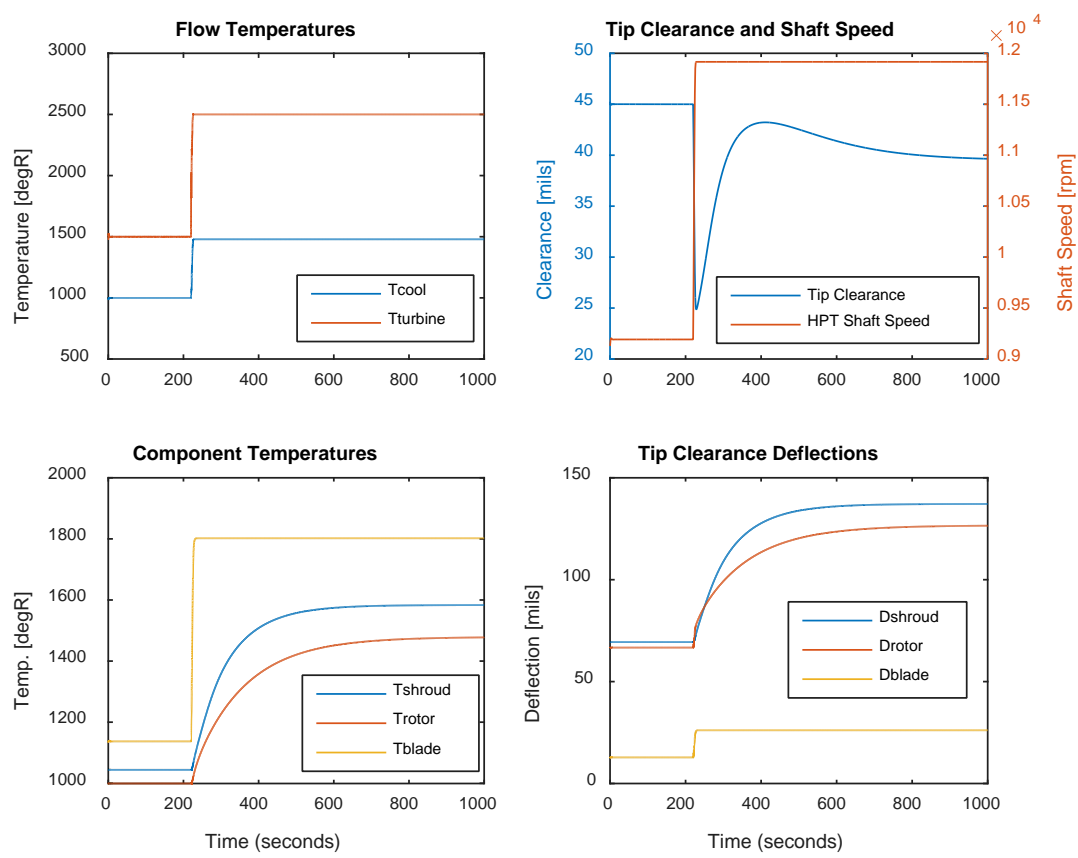

Figure 5. Deflections and clearance for simulated transient, ground idle to max power.

Internal temperature transients for the shroud and rotor are shown in Fig. 6 and Fig. 7, respectively. For each plot, temperature node states at the beginning of the simulation coincide with the $t=$ initial mark, and subsequent lines moving toward the top of the plots show the temperature distribution at 12 second intervals. In both cases, it can be seen that temperatures along the edges of the material are initially affected by the sudden rise in temperature. Once the penetration depth of the temperature transient has reached the center of the material, overall temperatures begin to rise. The profile of the rotor temperature keeps a fairly parabolic form that flattens out as it approaches steady state. The shroud temperature keeps a similar but skewed profile. The reason for this is because nodes 1 to 20 are a different material (abradable material) than the material (superalloy) in nodes 21 to 40 (see above). This abradable material acts as thermal barrier with lower thermal conductivity and convection coefficients than the superalloy material. The thermal barrier acts to dampen the heat transfer effects. Additionally in the shroud, the relatively large temperature rise of the turbine flow path shifts the temperature profile higher for the lower numbered nodes. It should be noted that although the node numbers are spaced evenly in Fig. 6, nodes 1-20 represent a thin coating on the material represented by nodes 21-40. 


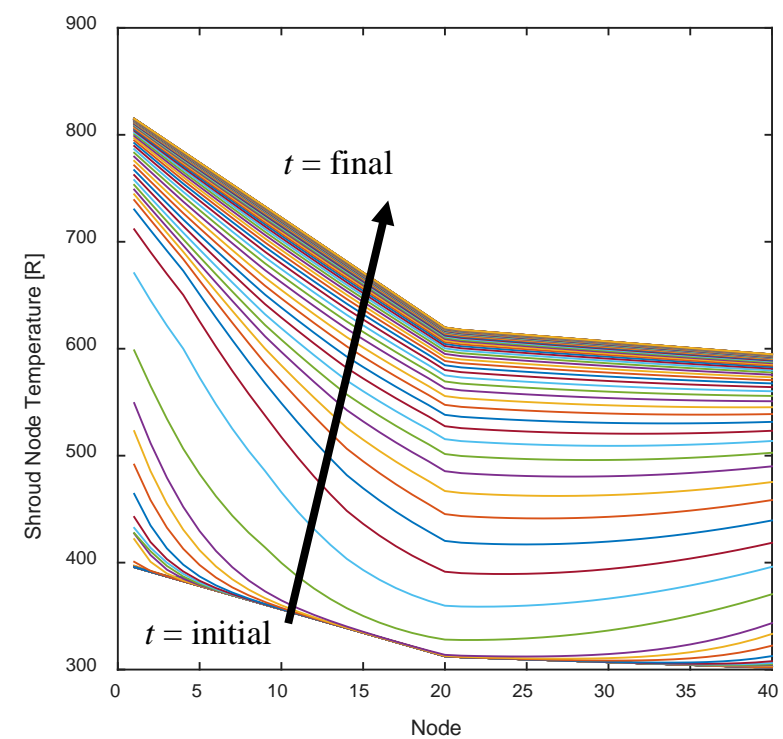

Figure 6. Shroud internal temperature transient.

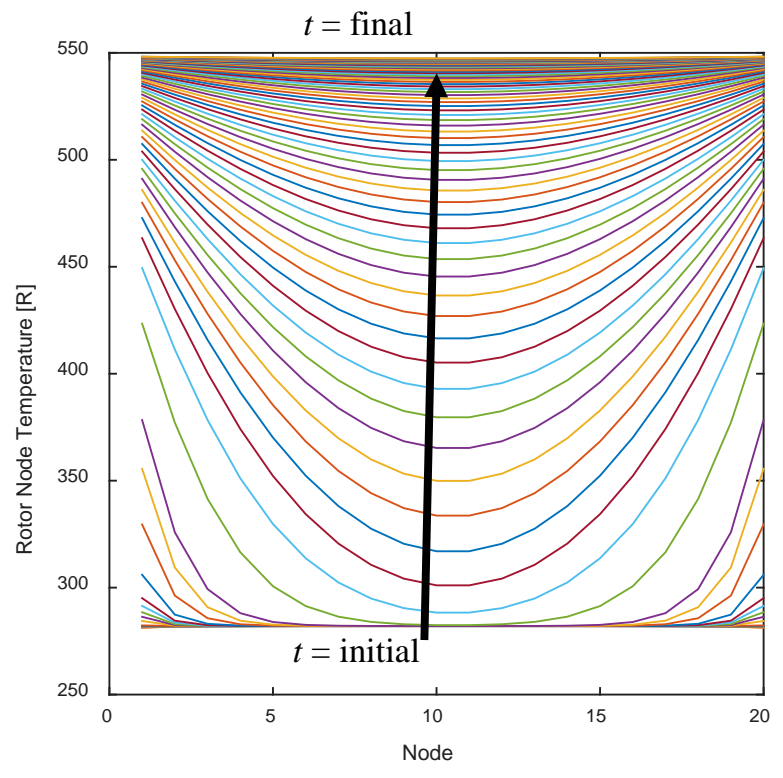

Figure 7. Rotor internal temperature transient.

Engine performance, comparing traces before and after the integration of tip clearance effects, is shown in Fig. 8. The traces demonstrate an engine acceleration at sea level static conditions (SLS) (200 s), followed by a reduction in throttle (400 seconds(s) to $600 \mathrm{~s}$ ) and increase in altitude ( $0 \mathrm{feet}(\mathrm{ft})$ to $35,000 \mathrm{ft}$ ) to cruise conditions. The intent of this trace is to capture clearance values for idle, the magnitude of clearance pinch point during an idle to full power transient, and the clearance at cruise conditions. Initially the engine operates at idle with tip clearance roughly the same as the nominal value. At $200 \mathrm{~s}$ the throttle is moved from idle to full power causing a large transient in engine operation and tip clearance. Values of exhaust gas temperature (EGT) and SFC are reduced by roughly $3 \%$ relative to the nominal value then slowly begins to rise, engine core speed $(N c)$ rises $0.7 \%$ as the turbine efficiency is increased, engine fan speed $(\mathrm{Nf})$ is reduced by about $1.75 \%$, and net thrust (Fnet) is reduced by about $4.5 \%$ due to the drop in $N f$. Reductions in performance values during the engine transient can be attributed to the $N c$ limiter remaining active longer as tip clearance values are reduced. This limiter is designed to prevent over-speed, and reacts to the higher core shaft speed values associated with the smaller tip clearance values, effectively damping out the response, as shown in Fig. 9 (with the baseline EPR controller equal to 1, acceleration limiter equal to 4, and $N c$ limiter equal to 6). This sluggish behavior could potentially be reduced by re-tuning the limiters in the control system; however, this type of controls exercise is beyond the scope of this paper and will not be explored here. Referring back to Fig. 8, once the engine speed transient is complete, the slower temperature transients begin to take effect and the engine control moves off the core speed and acceleration limiters. During the reduction in throttle and increase in altitude (400 s to $600 \mathrm{~s}$ and $0 \mathrm{ft}$ to 35,000 ft), the tip clearance shows an increase that coincides with large increases in SFC and EGT, small increases in Fnet and Nf, and decreases in static pressure at station 3 (Ps3) and Nc. Once the reduction in throttle is completed, tip clearance decreases resulting in an opposite effect. Signal noise observed in Fig. 8 traces for SFC, Fnet, and to a lesser extent Ps3 is the result of compounded simulated sensor noise and is independent of the tip clearance modeling. Overall, model trends determined in this simulation were found to be similar to those documented by Bringhenti and Barbosa, ${ }^{7}$ demonstrating realistic model behavior. It should be noted that the altitude change that occurs between $400 \mathrm{~s}$ and $600 \mathrm{~s}$ was performed more quickly than is realistic to reduce simulation runtime. This discrepancy was considered acceptable to the authors because it does not obscure the previously-stated objectives for the experiments. 

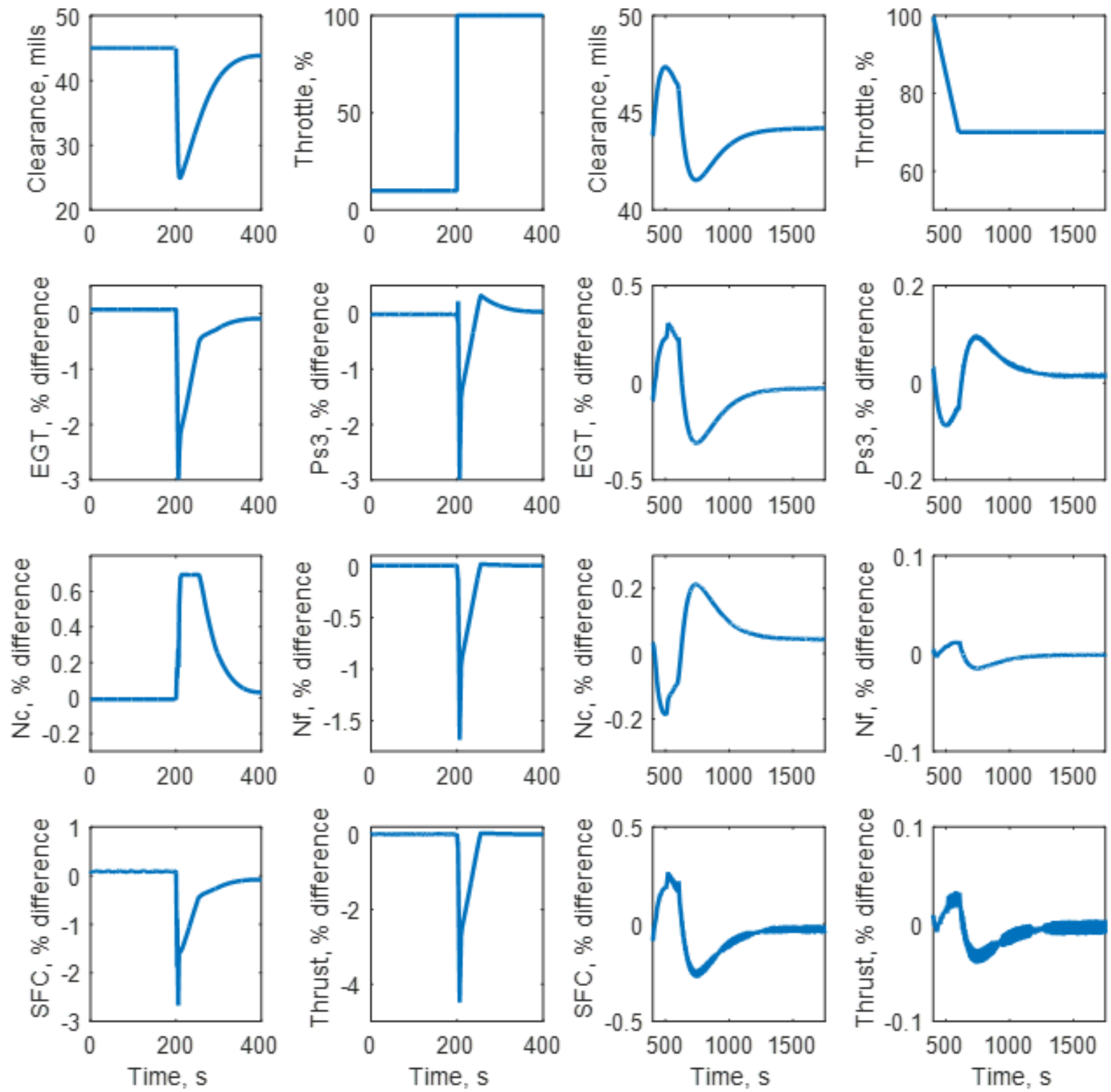

Figure 8. Un-integrated engine model and tip clearance integrated engine model difference traces for common performance parameters. Traces for an idle to full power throttle burst at SLS (200 s) appear in the left two columns and traces for a reduction in power from full to $70 \%$ and from $0 \mathrm{ft}$ to $35000 \mathrm{ft}(400 \mathrm{~s}$ to $600 \mathrm{~s}$ ) appear in the right two columns. 

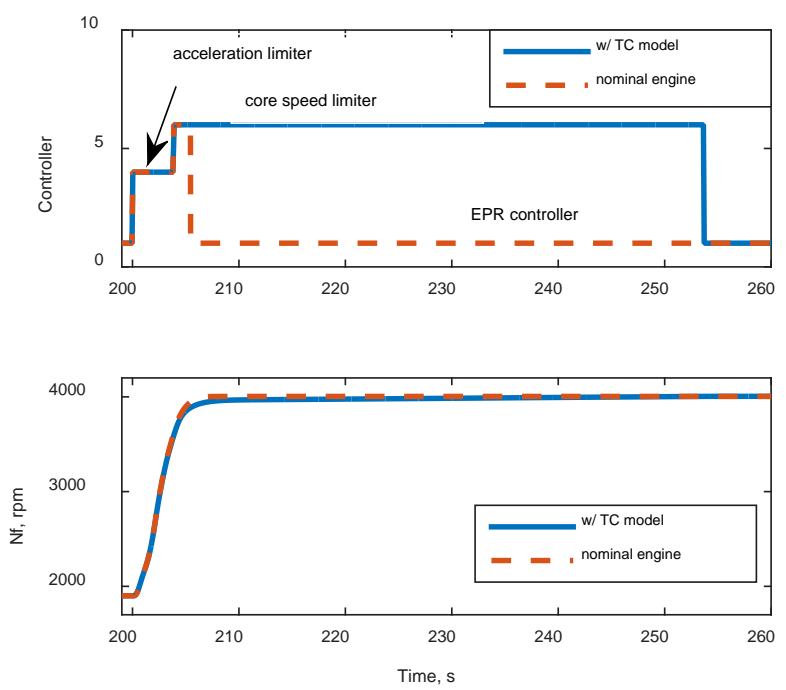
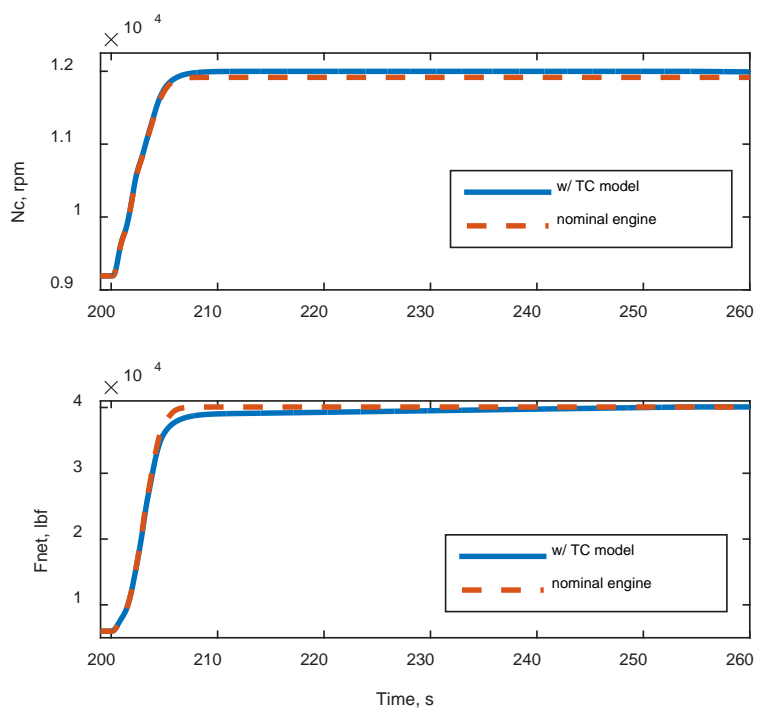

Figure 9. Controller, $N c, N f$, and Fnet for C-MAPSS40k with integrated tip clearance modeling (for throttle burst).

\section{Model Sensitivity Study}

For the previous section, the tip clearance model was run at a baseline configuration to demonstrate model viability. For this section, model inputs were adjusted to demonstrate clearance sensitivity to a range of alternate properties including, applied cooling temperature, surface convection characteristics, and material properties. The purpose of this study is to show the relationship between clearance and temperature for TCC purposes, and to better understand how the system may react to differences in materials or cooling flow architecture. Each example will show the same transient as detailed in the previous section, a quick SLS throttle movement from idle to full power at $200 \mathrm{~s}$ followed by a reduction in throttle and move to $35000 \mathrm{ft}$ starting at $400 \mathrm{~s}$ and ending at $600 \mathrm{~s}$, as shown in Fig. 8.

The first study is an analysis showing tip clearance's relationship with off baseline turbine shroud cooling flow temperatures. The cooling flow temperature was stepped from $20 \%$ lower than the baseline to $20 \%$ higher than the baseline and was directed only to the outer portion of the shroud. Looking at Fig. 10, it can be seen that increasing shroud cooling air temperature increases the tip clearance as warmer temperatures increase shroud size over the long term. Pinch point depth, relative to starting tip clearance, remains roughly the same from run to run, due to the fact these pinch point transients are dominated by rotor speed dynamics and not thermal dynamics. Results at cruise, after steady-state has been reached, show that changing shroud cooling flow temperature adjusts tip clearance with respect to the level at idle. Specifically, shroud cooling flow that is relatively hot raises the steady-state clearance, while relatively cool flow lowers clearance with respect to the idle clearance. This relationship shows that cooling flow temperature can be used to control the amount of tip clearance, effectively using relatively warm flow to increase clearance at idle and then adjusting the flow to be cooler after steady-state has been reached to close the gap and increase efficiency. An overview of performance parameters at steady-state idle and cruise operation is shown in Fig. 11. The figure highlights that SFC and EGT are increased, and Nc and Ps3 are decreased with larger clearance values. Differences in Fnet and $\mathrm{Nf}$ show only minor increases with larger clearances, which is to be expected because C-MAPSS40k is being controlled to EPR which is generally correlated to thrust. It can also be seen that difference values as a function of clearance are mostly linear across the regime. Comparing results at idle with those at cruise, the difference percent in SFC is greater at idle then it is at cruise signifying tip clearance has a larger effect on overall engine efficiency at lower power levels. 


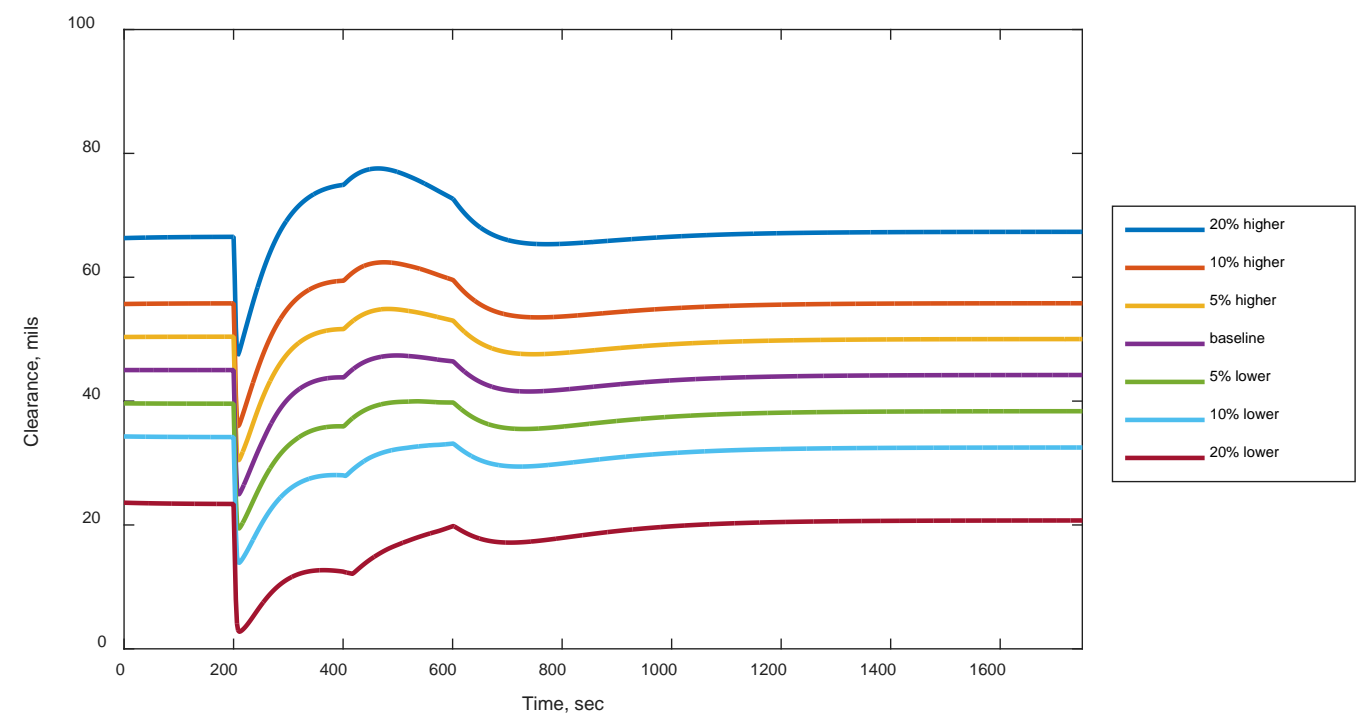

Figure 10. Clearance for an idle to full throttle burst followed by a reduction in power to cruise with various cooling flow temperatures.
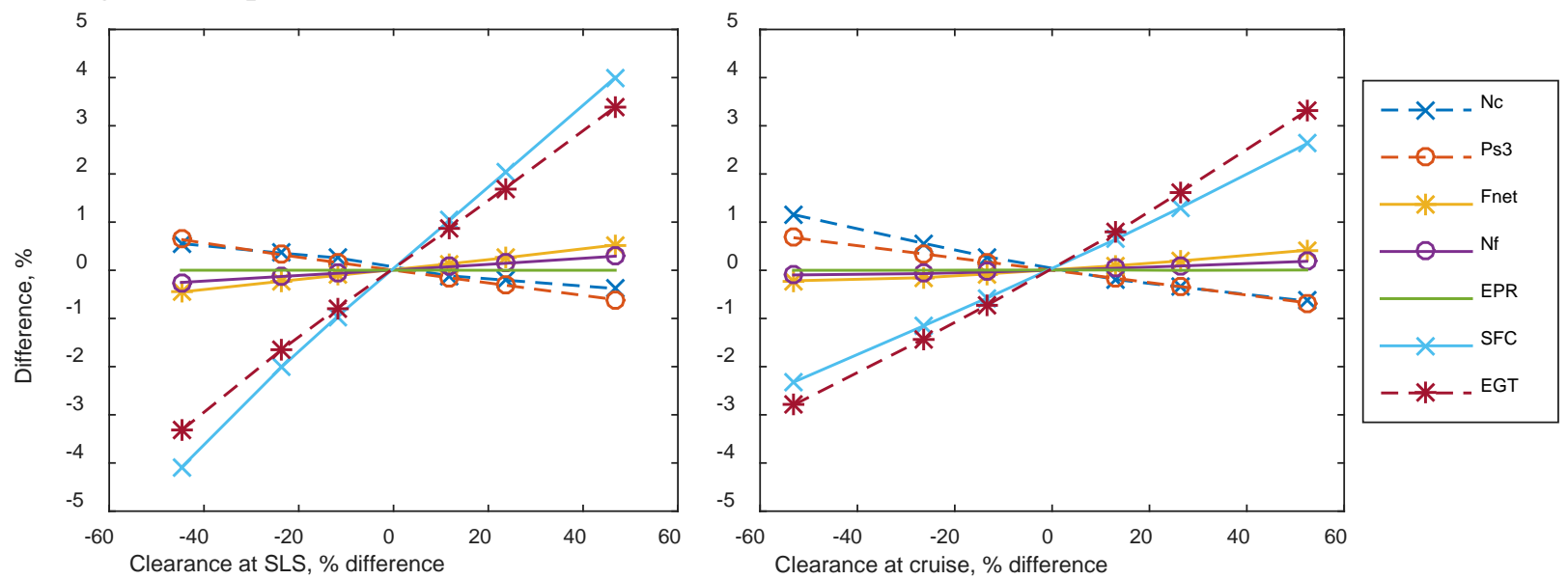

Figure 11. Common performance parameters for different clearance values at idle, SLS (left) and cruise, $35000 \mathrm{ft}$ (right).

The second study is an analysis of how changes in convection between flow streams and components can effect tip clearance. Heat transfer between the gas streams and the structural members is governed by the convection coefficient and can be determined by geometry, material properties, and mass flow. Because this modeling effort is non-specific (used for a generic engine), the geometry was assumed to be very simple and the convection coefficients were assumed to be constants. To determine the overall model sensitivity to differences in convection, the simulation was run with a high (two times baseline value) and low (one half baseline value) convection coefficient for each component. Each case was run using the transient detailed in the previous paragraph, with results shown in Fig. 12. The flow stream junctions considered for this analysis are the outer shroud/cooling flow, inner shroud/turbine flow, blade/turbine flow, and rotor/cooling flow. Steady-state differences show an increase in clearance by lowering the value of the convection coefficient for the outer shroud or raising the value of the convection coefficient for the inner shroud. Here, the maximum clearance difference is about 5 mils or thousands of an inch, from the baseline. Changes in blade convection coefficients show no deviation, and rotor convection coefficients show little to no effect on the turbine tip clearance. Additionally, pinch point clearance values (near 200 s) are mostly unaffected to the convection adjustment with a variation of only about 1 mil. 


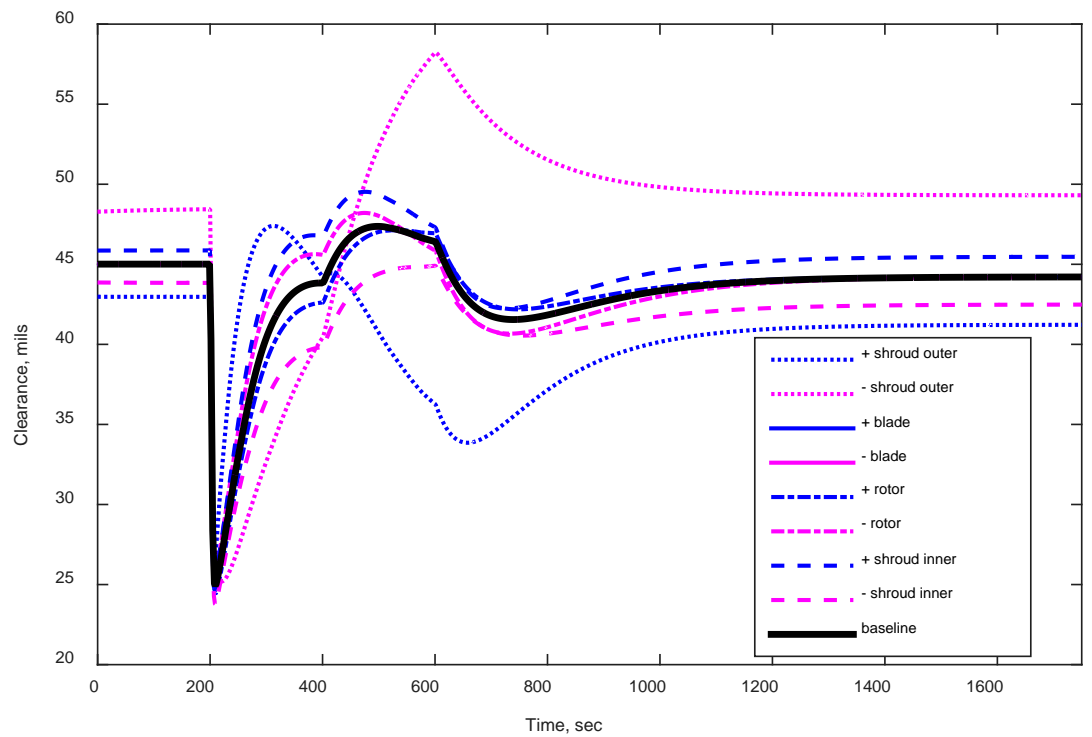

Figure 12. Turbine tip clearance with varying convection coefficients, +: baseline x 2, -: baseline / 2.

The final study is an analysis of how changes in material properties in the components can effect tip clearance. Heat transfer within the structural members is governed by the material properties that can change as a function of temperature. In the baseline model, the conduction coefficients and specific heats were assumed to be constant. To determine the overall model sensitivity to differences in material properties, the simulation was run with a high and low temperature set of material properties consistent with the temperature range each engine component may be exposed to during engine operation (conduction coefficient and specific heat two times and three times, respectively, at high temperatures; and one half and one third, respectively, at low temperatures). Each case was run using the transient detailed previously, as shown in Fig. 13. For this study it was assumed that the outer portion of the shroud and the rotor were composed of a similar material with properties that were comparable at any given time, as detailed in Ref. 4. Looking at the figure it can be seen that the largest steady-state changes in clearance (5 mils) come with adjusting the inner shroud material and the second largest effect (2 mils) comes from outer shroud/rotor material adjustment. Updating blade material properties made no difference to the overall clearance values with clearance values overlapping the baseline values. Pinch point clearance values (near $200 \mathrm{~s}$ ) vary up to 3 mils from the baseline with the adjusted inner shroud material properties. 


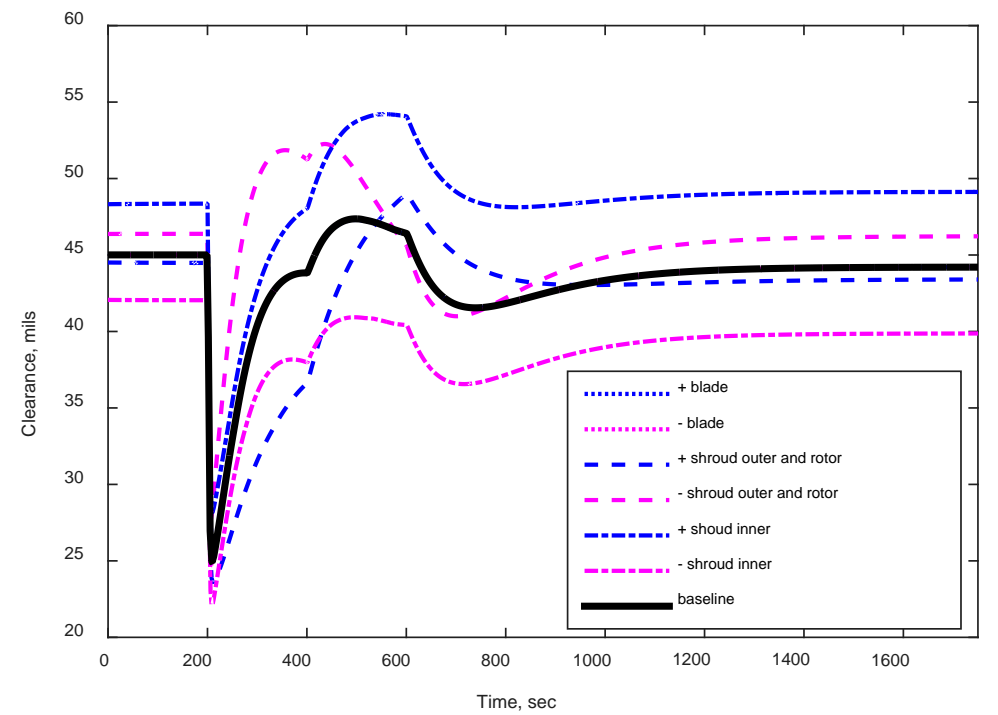

Figure 13. Turbine tip clearance with varying conduction coefficients and specific heats, +: baseline $k \times 2$ and $C_{p} \times 2$, -: baseline $k$ / 2 and $C_{p} / 3$.

By looking at Fig. 10 and Fig. 11 and assuming a 1\% change in any performance criterion is significant it can be determined that any change in modeling behavior that would result in a 5 mils adjustment in clearance is also significant. This generality can then be extended to determine that changes in shroud cooling temperature of $5 \%$ or greater, in outer shroud conduction on the order of two times, or in material properties of the inner shroud on the order of two times for $k$ and three times for $C_{p}$ are also significant. Changes of significance result in clearances that may need to be taken into account by the model and/or may be exploited for tip clearance control purposes. The above criterion can also be used to determine that none of the above methods may be used to make significant adjustments to the pinch point relative to clearance values at idle.

\section{Future Work}

In the previous section, sensitivity studies were conducted to determine the overall effect of changes in shroud cooling flow temperature and component material properties. These studies found that each can have a significant impact on turbine tip clearance. Suggestions for future work that could contribute to expanding the body of knowledge in this area are: the addition of a temperature dependent material properties model, the addition of a convection coefficient model that varies with engine operation conditions, the exploration of tip clearance control schemes that make use of shroud temperature adjustment to minimize the pinch point relative to cruise conditions, studies exploring the relationship of tip clearance with engine deterioration, and research regarding alternate methods of pinch point reduction.

\section{Conclusions}

Managing tip clearance during aircraft engine operation can have the benefit of increasing engine efficiency and lowering operating temperatures. This paper discusses the development of a tip clearance model that was integrated with a high fidelity engine model to create a platform for active tip clearance control research. The integrated engine model demonstrated realistic tip clearance behavior, with the response dominated by fast acting centrifugal forces and slower acting thermal forces. A sensitivity study looked into how cooling temperature, component convective relationships, and material properties can affect tip clearance and how tip clearance affects engine performance. These studies show how adjustments in shroud cooling temperature, outer shroud convection effects, and inner shroud material properties can have a significant effect on steady-state tip clearance. The performance study also confirms significant reductions in exhaust gas temperature and specific fuel consumption as turbine tip clearance is reduced. Results from the studies were then leveraged to identify future work to be done to improve model fidelity and additional research areas. 


\section{Acknowledgments}

The authors would like to thank the NASA Advanced Air Transport Technology (AATT) project for funding this work.

\section{References}

${ }^{1}$ Lattime, S.B., Steinetz, B.M., “Turbine engine clearance control systems: current practices and future directions,” American Control Conference, 2001.

${ }^{2}$ Hamed, A., Tabakoff, W., Wenglarz, R., “Erosion, Deposition, and Their Effect on Performance,” Progress in Astronautics and Aeronautics, Vol. 243, AIAA, VA, 2014, Chap. 14.

${ }^{3}$ Melcher, K.J., “Controls Considerations for Turbine Active Clearance Control”, NASA/CP-2004-212963/Vol. 1.

${ }^{4}$ Krypuros, J.A., Melcher, K.J., “A Reduced Model for Prediction of Thermal and Rotational Effects on Turbine Tip Clearance,” NASA/TM-2003-212226.

${ }^{5}$ Holeski, D.E., Futral, S.M, "Effect of Rotor Tip Clearance on the Performance of a 5-inch Single-Stage Axial-Flow Turbine,” NASA TM X-1757, 1969.

${ }^{6}$ Wiseman, M.W., Guo, T-H., “An investigation of life extending control techniques for gas turbine engines,” Proceedings of the American Control Conference, 2001.

${ }^{7}$ Bringhenti, C., Barbosa, J.R., “Effects of Turbine Tip Clearance on Gas Turbine Performance,” Proceedings of ASME Turbo Expo 2008: Power for Land, Sea and Air, GT2008-50196, Berlin, Germany, June, 2008.

${ }^{8}$ DeCastro, J.A., Melcher, K.J., “A Study on the Requirements for Fast Active Turbine Tip Clearance Control Systems,” AIAA2004-4176, AIAA Joint Propulsion Conference \& Exhibit, Fort Lauderdale, Florida, July, 2004.

${ }^{9}$ May, R.D., Csank, J., Lavelle, T.M., Litt, J.S., and Guo, T-H., “A High-Fidelity Simulation of a Generic Commercial Aircraft Engine and Controller,” AIAA-2010-6630, AIAA Joint Propulsion Conference, Nashville, TN, July, 2010.

${ }^{10}$ Csank, J., May, R.D., Litt, J.S., Guo, T-H., “Control Design for a Generic Commercial Aircraft Engine,” AIAA-2010-6629, AIAA Joint Propulsion Conference \& Exhibit, Nashville, TN, July, 2010.

${ }^{11}$ Incropera, F.P., DeWitt, D.P., “Fundamentals of Heat and Mass Transfer,” John Wiley \& Sons, New York, NY, 2002.

${ }^{12}$ Crank, J., Nicolson, P., "A Practical Method for Numerical Evaluation of Solutions of Partial Differential Equations of the Heat Conduction Type,” Advances in Computational Mathematics, Dec. 1996, Vol 6, Issue 1, pp. 207-226.

${ }^{13}$ Chapman, J.W., Lavelle, T.M., May, R.D., Litt, J.S., Guo, T.H., “Toolbox for the Modeling and Analysis of Thermodynamic Systems (T-MATS) User’s Guide,” NASA/TM-2014-216638, January 2014.

${ }^{14}$ Baskharone, E.A., "Principles of Turbomachinery in Air-Breathing Engines," Cambridge Aerospace Series, Cambridge University Press, 2006. 